\title{
Synthesis, oral bioavailability evaluation and antiplatelet aggregation activity of three derivatives of 3,4-dihydroxyacetophenone
}

\author{
NA SUN, MEI QU, MENGYU WANG, YANNA LV, LIUYA WEI and YU DUAN \\ School of Pharmacy, Weifang Medical College, Weifang, Shandong 261053, P.R. China
}

Received March 27, 2019; Accepted November 21, 2019

DOI: $10.3892 /$ ijmm.2020.4469

\begin{abstract}
Dihydroxyacetophenone (DHAP) exerts therapeutic effects on cardiovascular disease and pulmonary heart disease. However, it is not utilized as a clinical drug due to its rapid metabolism, short-acting effect and low oral bioavailability. In the present study, three derivatives of DHAP, including 4-acetyl-1,2-phenylene dipropionate (APDP), 1-(3-hydroxy-4-phenoxy-phenyl)-ethanone (HPPE) and a polymer derivative, PEG-DHAP, were synthesized via the esterification or etherification of hydroxyls at C3 and C4, which are the prime metabolism sites of DHAP. The physicochemical properties, pharmacokinetic and antiplatelet aggregation activities of these derivatives were measured to determine whether they can improve the defects of DHAP. The results revealed that APDP and HPPE exhibited markedly lower water solubility and higher oil-water partition coefficients compared to DHAP. APDP rapidly transformed into DHAP in vivo and in vitro, indicating that there were no significant differences in the values of mean residence time in vivo [MRT $\left._{(0-t)}\right]$, half-life $\left[\mathrm{t}_{1 / 2}\right]$, oral bioavailability and antiplatelet aggregation activity in vivo and in vitro between the two agents. HPPE partially released DHAP for a period of time following oral administration. PEG-DHAP contained $12.47 \%$ DHAP dispersed into nanoparticles with a mean particle size of $260.90 \mathrm{~nm}$. This was administered in aqueous solution and was demonstrated to release DHAP slowly following oral administration. These two derivatives significantly prolonged the in vivo acting time and increased the oral bioavailability of DHAP: Following intragastric administration, their $\mathrm{MRT}_{(0-\mathrm{t})}$ and $\mathrm{t}_{1 / 2}$ values were 3.55-11.47- and 6.63-11.25-fold higher compared with those of DHAP, respectively. Additionally, their relative bioavailability was 394.79 and $331.88 \%$, respectively, and they exhibited
\end{abstract}

Correspondence to: $\mathrm{Dr} \mathrm{Yu}$ Duan, School of Pharmacy, Weifang Medical College, 7166 West Baotong Road, Weifang, Shandong 261053, P.R. China

E-mail: duanyu@wfmc.edu.cn

Key words: 3,4-dihydroxyacetophenone, esterification, etherification, pharmacokinetic, oral bioavailability, antiplatelet aggregation longer acting times of significant antiplatelet aggregation activity. The results of the present study may thus provide a reference for the development of DHAP as an oral drug. Furthermore, the results if this study may prove to be useful to researchers addressing the issue of the poor bioavailability of phenolic drugs.

\section{Introduction}

In 1971, 3,4-dihydroxyacetophenone (DHAP; also referred to as Qingxintong) was isolated from the leaves of Ilex Pubescens Hook et Arnvar glaber Chang, which is a traditional Chinese herb that improves blood circulation (1). Since then, several studies have demonstrated that DHAP effectively improves the clinical symptoms of patients with angina pectoris, pulmonary heart disease and pregnancy-induced hypertension (2-12). Clinical and animal pharmacological studies have revealed that DHAP exerts antiplatelet aggregation effects (13-18), inhibits cyclooxygenase (18), reduces the fluidity of platelet membranes (19) and inhibits thrombus formation by regulating the ratio of thromboplastin to thromboplastin inhibitor in the human umbilical vein endothelium (20). DHAP also dredges microcirculation (6,7,21-23). It has been demonstrated that DHAP significantly enhances the delayed $\mathrm{K}^{+}$current of vascular smooth muscle and promotes the opening of $\mathrm{K}^{+}$channels, thereby relaxing smooth muscle, relaxing the coronary arteries and improving myocardial function (21,24). Additionally, DHAP has been shown to correct the imbalance of thromboxane $\mathrm{TAX}_{2} /$ prostacyclin $\mathrm{PGI}_{2}$ and the imbalance of $\mathrm{NO} /$ endothelin in maternal and uterine placental vascular beds, thereby improving uterine and placental blood circulation in patients with pregnancy-induced hypertension $(9,25,26)$. DHAP also exerts anti-inflammatory effects and affects the occurrence and development of atherosclerotic inflammatory responses through various mechanisms (27-32).

Although clinical studies have demonstrated that DHAP exerts therapeutic effects on cardiovascular and pulmonary heart disease, it is not administered as a clinical drug due to it being a short-acting compound that is eliminated from the body relatively quickly. Pharmacokinetic studies in animal experiments have revealed that DHAP is absorbed rapidly and eliminated quickly, that the absorption half-life and the elimination half-life of intramuscular (i.m.) injection and 
intragastric (i.g.) administration are in the range of 0.05-0.07 and $0.14-0.29 \mathrm{~h}$, respectively, and that it exhibits low oral bioavailability in the range of 9.16-22.05\% due to severe first-pass metabolism $(33,34)$. Since the in vivo acting time of DHAP is very short, frequent administration is required to maintain an acceptable blood concentration to achieve its clinically therapeutic purpose. These shortages significantly impede the clinical application of DHAP.

The chemical structure of DHAP is presented in Fig. 1. DHAP is soluble in water and has a low molecular weight (152 $\mathrm{g} / \mathrm{mol})$, meaning its absorption and distribution is rapid and primarily occurs via passive diffusion across intestinal epithelial cells. Similar to most phenolic and catechol containing drugs, DHAP tends to be rapidly cleared by sulfation, glucuronidation and 3-O-methylation in vivo (35). Modifying the sites of metabolism and phenolic hydroxyls of DHAP, as is the case for esterification and etherification, affects certain pharmacokinetic processes and oral bioavailability. In the present study, three derivatives of DHAP were prepared via modification of C-3 or C-4 phenol hydroxyls. 4-Acetyl-1,2-phenylene dipropionate (APDP) was prepared by esterification of C-3 and C-4 phenol hydroxyls, 1-(3-hydroxy-4-phenoxy-phenyl)-ethanone (HPPE) was prepared via etherification of the C-4 phenol hydroxyl, and the polymer derivative (PEG-DHAP) was prepared by attaching DHAP to polyethylene glycol 2000 (PEG 2000) via ester bonds. Ester and ether bonds were selected as they can be cleavage-catalyzed by enzymes in vivo, after which the molecular structure of DHAP may be recovered. Compared with DHAP, APDP and HPPE contain hydrophobic hydrocarbon groups, which confer lower water solubility and higher oil-water distribution coefficients. For the polymer derivative, PEG-DHAP, the PEG barriers protect DHAP against rapid clearance in vivo, as only free DHAP diffusing out of the PEG barriers may be cleared. The pharmacokinetic processes of these derivatives differ from those of DHAP, which is expected to a certain extent.

In the present study, certain physicochemical properties closely associated with absorption and distribution were assessed. These included the water solubility and oil-water partition coefficients of DHAP, APDP and HPPE, the mean particle size of PEG-DHAP in aqueous solution and the release of three DHAP derivatives in vitro and in vivo. The concentrations of the three derivatives in rat blood following a single oral administration were measured. The pharmacokinetic parameters were compared with those of DHAP to assess whether these derivatives may prolong in vivo acting time and improve oral bioavailability. The antiplatelet aggregation was then selected as an indicator of activity to discuss the bioactivity of these derivatives.

\section{Materials and methods}

Chemicals. DHAP $(\geq 98 \%)$ was obtained from Jinan Luxin Chemical Technology Co., Ltd. Propionyl chloride and succinyl chloride, each of analytical grade, were purchased from Aladdin Reagents Co., Ltd. Benzyl bromide and N,N-dimethylformamide (DMF), each of analytical grade, were purchased from Sinopharm Chemical Reagent Co., Ltd. and re-evaporated prior to use. Triethylamine (TEA) and acetone, both of analytical grade, were purchased from Tianjin Kemiou Chemical Reagent Co., Ltd. and dehydrated prior to use. PEG 2000, an experimental reagent, was purchased from Tianjin Damao Chemical Reagent Factory. Adenosine diphosphate (ADP), a biochemical reagent, was purchased from Sigma-Aldrich; Merck KGaA and dissolved in physiological saline prior to use. Other chemicals were purchased from local chemical reagent suppliers.

Animals. Male rabbits and male/female Wistar rats (weight, 250-300 g) were purchased form the Experimental Animal Center of Shandong Lukang Pharmaceutical Co. (license nos. 0017075 and 0010061, respectively). The experimental procedures were approved by the Animal Experimentation Ethics Committee of Weifang Medical College and were conducted in accordance with the guidelines of the National Health and Medical Research Council of China for the care and use of animals. Pior to the experiments, the rabbits and rats were reared for 1 week in an environment with a temperature of $25^{\circ} \mathrm{C}$ and a humidity of $40-70 \%$. They were fasted only from food but were still provided with water for $12 \mathrm{~h}$ prior to the experiment. For the determination of the behaviors of derivatives releasing DHAP in the in vitro and in vivo experiments, 21 rats were used, and blood was obtained from the fundus venous plexus after the rats inhaled isoflurane for anesthesia. The dose of isoflurane used was 3-4\% for induction and $2-2.5 \%$ for maintenance, and the oxygen flow rate was $500-700 \mathrm{ml} / \mathrm{min}$. These rats continued to be raised for 2 weeks for other uses. In the pharmacokinetic experiments, 36 rats were used, and blood was obtained from the fundus venous plexus after the rats inhaled isoflurane for anesthesia. The dose of isoflurane used was the same as the one described above for the determination of derivatives releasing DHAP. These rats were sacrificed by cervical dislocation after the end of the in vivo experiment. For the in vitro antiplatelet aggregation activity experiments, 3 rabbits were used. The rabbit ear vein was injected with a concentration of $3 \%$ sodium pentobarbital solution for anesthesia at a dose of $30 \mathrm{mg} / \mathrm{kg}$. Following the collection of approximately $80-100 \mathrm{ml}$ of blood from the carotid artery, the rabbit ear veins were injected with air to terminate life. For the in vivo antiplatelet aggregation activity experiments, 54 rats were used and were anesthetized with sodium pentobarbital $(3 \%)$ via an intraperitoneal injection at a dose of $40 \mathrm{mg} / \mathrm{kg}$. The chest of the animals was then opened and $5 \mathrm{ml}$ blood were obtained from the heart, and the rats were subsequently decapitated.

Synthesis of APDP. DHAP (2.01 g; $13.25 \mathrm{mmol})$ was dissolved in $140 \mathrm{ml}$ acetone, followed by the addition of TEA (4.60 ml; $33.00 \mathrm{mmol})$ and stirring at room temperature for $1 \mathrm{~h}$. Propionyl chloride $(3.00 \mathrm{ml} ; 34.37 \mathrm{mmol})$ was dissolved in $10 \mathrm{ml}$ acetone and slowly dropped into the DHAP solution on ice. The solution was then stirred at room temperature for $8 \mathrm{~h}$. The reaction solution was evaporated to dryness under reduced pressure, and the remaining residue was dissolved in ethyl acetate. The solution was washed successively with water and saturated sodium chloride aqueous solution. The resultant ethyl acetate layer was dried over anhydrous magnesium sulfate overnight. The solution was filtered and the 


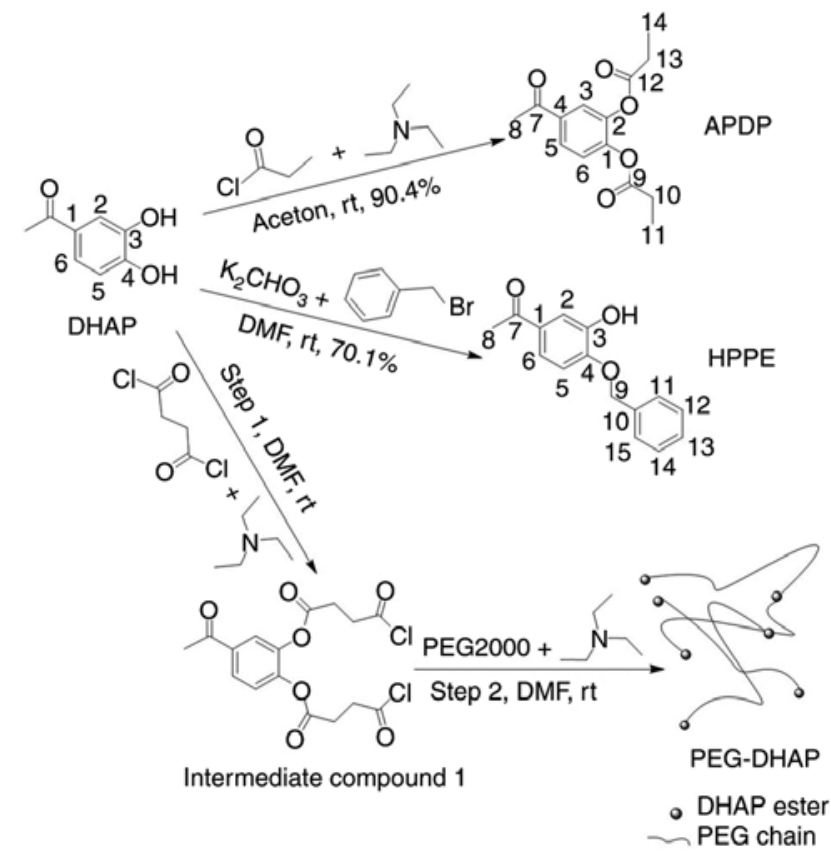

Figure 1. Schematic of the synthesis of the derivatives. DHAP, 3,4-dihydroxyacetophenone; APDP, 4-acetyl-1,2-phenylene dipropionate; HPPE, 1-(3-hydroxy-4-phenoxy-phenyl)-ethanone.

filtrate was evaporated to dryness under reduced pressure to create a colorless powder.

Synthesis of HPPE.DHAP $(2.54 \mathrm{~g} ; 16.70 \mathrm{mmol})$ and anhydrous potassium carbonate $(2.31 \mathrm{~g} ; 16.72 \mathrm{mmol})$ were sequentially added into $42 \mathrm{ml}$ DMF under a nitrogen atmosphere and stirred at room temperature for $1 \mathrm{~h}$. Benzyl bromide $(1.99 \mathrm{ml}$; $16.74 \mathrm{mmol}$ ) was slowly dropped into the aforementioned mixture in an ice bath. The mixture was subsequently stirred at room temperature for $90 \mathrm{~min}$. The mixture was filtered and the filtrate was diluted with 5-fold volumes of ethyl acetate, after which time the solution was washed sequentially with water and saturated sodium chloride aqueous solution. The ethyl acetate phase was dried over anhydrous magnesium sulfate overnight. The solution was filtered, and the filtrate was evaporated to dryness under reduced pressure to create a light-yellow powder.

Synthesis of PEG-DHAP. DHAP (1.00 g; $6.60 \mathrm{mmol}$ ) and TEA $(2.10 \mathrm{ml} ; 15.12 \mathrm{mmol})$ were dissolved in $50 \mathrm{ml} \mathrm{DMF}$ and the solution was stirred at room temperature for $1 \mathrm{~h}$. Succinyl chloride $(1.7 \mathrm{ml} ; 14.53 \mathrm{mmol})$ was dropped into the solution slowly in an ice bath. The solution was subsequently stirred at room temperature for $8 \mathrm{~h}$ to yield an intermediate (product 1 ) solution. PEG 2000 (6.63 g; $3.32 \mathrm{mmol})$ and TEA $(1.06 \mathrm{ml}$; $7.64 \mathrm{mmol}$ ) were dissolved in $50 \mathrm{ml}$ DMF, after which time the solution was stirred at room temperature for $1 \mathrm{~h}$. Intermediate (product 1) solution was dropped into the second solution slowly. The resultant solution was then stirred at room temperature for $24 \mathrm{~h}$. After the reaction had taken place, the solution was concentrated under reduced pressure and dialyzed in a dialysis bag with a molecular weight of 1,500 against deionized water for 3 days. The samples were then freeze-dried to create a light brown powder.
Water solubility determination. Water solubility was determined according to the method previously described by Montenegro et al (36). An excess quantity of compound was weighed into a glass tube containing $2 \mathrm{ml}$ of water, and the tube was sealed with a Teflon-lined cap. The mixture was stirred with a magnetic stirrer for $24 \mathrm{~h}$ at room temperature and then filtered. The quality of the compound in its saturated solution was determined via HPLC.

Oil-water partition coefficient determination. The oil-water partition coefficient was determined using the classical shake flask method (37). The quality of the compound in solution was determined via HPLC.

Content of DHAP in PEG-DHAP. PEG-DHAP (30 mg) was dissolved in $2 \mathrm{ml}$ distilled water and sealed in a dialysis bag (Spectrumlabs, MD31, MW 500-1,000). The dialysis bag was incubated in $50 \mathrm{ml}$ aqueous hydrochloric acid solution $(\mathrm{pH} 2)$ at room temperature. Hydrochloric acid solution $(0.5 \mathrm{ml})$ was sampled and the quality of DHAP was determined via HPLC. After each sampling, $0.5 \mathrm{ml}$ hydrochloric acid solution $(\mathrm{pH} 2)$ were added. The measurement continued until the quality of DHAP no longer changed.

HPLC conditions. A 1260 Infinity chromatography system (Agilent Technologies, Inc.) was used. It was equipped with a DAD detector (G1315D VL; Agilent Technologies, Inc.), a column (Hypersil ODS2; 250x4.60 mm; $5 \mu \mathrm{m}$; Illit) and an injector (2PS/6PT; Mon Inj; 600 bar; Agilent Technologies, Inc.). The sample volume was $20 \mu \mathrm{l}$ and the flow rate was $1 \mathrm{ml} / \mathrm{min}$. The eluent was composed of methanol and water, with a volume ratio of 60:40. The elution was performed at room temperature and was detected at a wavelength of $275 \mathrm{~nm}$.

Particle size determination of PEG-DHAP in aqueous solution. PEG-DHAP powder was dissolved in distilled water by ultrasonication to obtain a $0.5 \mathrm{mg} / \mathrm{l}$ PEG-DHAP solution. Particle size and distribution were determined using the Malvern Zetasizer Nano Series 90 (Malvern Instruments, Ltd.

Determination of the behaviors of derivatives releasing DHAP in vitro and preparation of plasma samples. Rat blood $(0.5 \mathrm{ml})$ was collected into a centrifuge tube containing $55 \mu 13.8 \%$ $(\mathrm{w} / \mathrm{v})$ sodium citrate solution via the fundus venous plexus and centrifuged at $1,425 \mathrm{x}$ g for $10 \mathrm{~min}$ at room temperature. The supernatant plasma was then separated. Subsequently, $100 \mu 1$ plasma were precisely obtained and $10 \mu \mathrm{l}$ methanol solution of phenol (internal standard) with $10 \mu \mathrm{l}$ methanol solution of derivative were added. The mixture was then vortexed and $400 \mu \mathrm{l}$ ethyl acetate were added. Following a second vortex and centrifugation at $1,425 \mathrm{x}$ g for $10 \mathrm{~min}$ at room temperature, the supernatant was collected. Ethyl acetate $(200 \mu \mathrm{l})$ was further added to the precipitate, which was vortexed and centrifuged at $1,425 \mathrm{x} g$ for $10 \mathrm{~min}$ at room temperature. The supernatant was collected and combined with that obtained previously. The combined supernatants were then dried with nitrogen and dissolved in $100 \mu \mathrm{l}$ mobile phase for HPLC analysis. For comparison, the methanol solutions of the derivatives were also analyzed by HPLC. 
The percentage of derivative converted to DHAP was calculated using internal standard correction factor method as the following formula.

$$
\frac{\overline{\overline{A_{\text {DHAP }}}}}{\overline{\overline{\overline{A_{\text {phenol }}}}}} \times 100 \%
$$

where $\overline{A_{D H A P}}$ is the average value of the peak area of DHAP, $\overline{A_{\text {phenol }}}$ is the average value of the peak area of internal standard phenol and $\overline{A_{\text {derivative }}}$ is the average value of the peak area of derivatives.

Determination of the behaviors of derivatives releasing DHAP in vivo. The rats were randomly divided into 3 groups (APDP, HPPE and PEG-DHAP) with 6 rats in each. The derivatives were dissolved in physiological saline to prepare a solution or suspension. The doses used for the rats (i.g.), as recommended by previous studies $(33,34)$, were $0.79 \mathrm{mmol} / \mathrm{kg}$ APDP and HPPE, and $0.55 \mathrm{~g} / \mathrm{kg}$ PEG-DHAP (containing $0.45 \mathrm{mmol}$ DHAP). At 10 min after the administration, $0.5 \mathrm{ml}$ blood were collected via the fundus venous plexus. The preparation of the plasma sample was the same as the aforementioned, apart from the fact that a methanol solution of derivative was added.

HPLC conditions. The aforementioned HPLC conditions were used, apart from the elution and detection wavelength. The elution was a linear gradient of methanol and water: $0 \mathrm{~min}$ $(40: 60 \mathrm{v} / \mathrm{v}), 5.5 \mathrm{~min}(60: 40 \mathrm{v} / \mathrm{v})$ and $15 \min (40: 60 \mathrm{v} / \mathrm{v})$. The detection wavelength was $260 \mathrm{~nm}$.

Preparation of plasma for antiplatelet aggregation activity. Blood was collected from the carotid artery of rabbits or the heart of rats. Sodium citrate solution $(3.8 \% \mathrm{w} / \mathrm{v})$ was used as the anticoagulant and was mixed with blood at a volume ratio of 1:9. Platelet-rich plasma (PRP) was obtained by centrifugation at $285 \mathrm{x} \mathrm{g}$ for $10 \mathrm{~min}$ at room temperature. Platelet-poor plasma (PPP) was obtained by centrifugation of the remaining blood at $1,425 \mathrm{x}$ g for $15 \mathrm{~min}$ at room temperature. The number of platelets in PRP was adjusted using PPP to $\sim 5 \times 10^{8}$ cells $/ \mathrm{ml}$.

Antiplatelet aggregation activity of rabbits in vitro. PRP (300 $\mu \mathrm{l}$ ) was pre-incubated for $3 \mathrm{~min}$ at $37^{\circ} \mathrm{C}$ with various concentrations of compound that were pre-dissolved in dimethyl sulfoxide (DMSO). The final concentration of DMSO in PRP was $<1.0 \%(\mathrm{v} / \mathrm{v})$ to eliminate a false positive result. Platelet aggregation was subsequently stimulated by ADP (final concentration in PRP, $10 \mu \mathrm{mol} / \mathrm{l})$. Aggregation was monitored using a platelet aggregometer (LBY-NJ4A; Precill) at a constant stirring speed of $1,200 \mathrm{rpm}$, and aggregation rates were recorded for $5 \mathrm{~min}$ to determine the percentage aggregation. The aggregation inhibition rate (AIR) was calculated using the following formula:

$$
\operatorname{AIR}(\%)=1-\frac{A R_{\text {compoutal }}}{A R_{\text {vehicle }}} \times 100 \%
$$

where AR stands for the aggregation rate.

Antiplatelet aggregation activity in vivo in rats. Wistar rats were randomly divided into 9 groups (the vehicle,
DHAP10, APDP10, HPPE10, PEG-DHAP10, DHAP40, APDP40, HPPE40 and PEG-DHAP40), 6 in each group. The compounds were dissolved in physiological saline to prepare a solution or suspension. The doses used for the rats (i.g.) were $0.79 \mathrm{mmol} / \mathrm{kg}$ DHAP, APDP and HPPE, and $0.55 \mathrm{~g} / \mathrm{kg}$ PEG-DHAP (containing $0.45 \mathrm{mmol}$ DHAP). The rats in the vehicle group were administered the same volume of i.g. physiological saline. After 10 and $40 \mathrm{~min}$ of administration, $5 \mathrm{ml}$ blood were collected.

PRP was pre-incubated for $3 \mathrm{~min}$ at $37^{\circ} \mathrm{C}$ and then stimulated by ADP. The aforementioned procedures were used to assess the concentration of ADP and for the measurement of platelet aggregation.

Statistical analysis for antiplatelet aggregation activity. Data are presented as the means \pm SD. Statistical significance was determined by an unpaired t-test or two-way ANOVA followed by Tukey's multiple comparisons test (SPSS 9.0 for Windows; SPSS, Inc.). A value of $\mathrm{P}<0.05$ was considered to indicate a statistically significant difference with $\alpha=0.05 \%$. In the in vitro experiments, each drug concentration group was independently determined 4 times. In the in vivo experiments, there were 6 rats in each group, and the blood of each rat was independently determined 4 times.

HPLC conditions for the measurement of blood concentration. The HPLC conditions were same as the aforementioned, apart from the detection wavelength, which was set to $275 \mathrm{~nm}$ for DHAP and $246 \mathrm{~nm}$ for HPPE.

Specific investigation. The internal standard and DHAP were added to both blank plasma and plasma samples, and these plasma samples were measured using HPLC to assess the specificity of chromatographic conditions.

Preparation of DHAP and HPPE standard curves in plasma. Serial concentrations of DHAP or HPPE standard solution were prepared using methanol as a solvent. Standard solution $(10 \mu \mathrm{l})$ and methanol solution of phenol (internal standard; $10 \mu \mathrm{l}$ ) were added to $100 \mu \mathrm{l}$ blank plasma. Each mixture was processed as described above in the paragraph entitled 'Determination of the behaviors of derivatives releasing DHAP in vitro and preparation of plasma samples'. The peak area ratio (DHAP or HPPE vs. internal standard) of each sample was determined by HPLC and plotted against the concentration. Linear regression was performed to produce the standard curve.

Recovery and precision control. High, medium and low concentrations of DHAP or HPPE standard solutions were added to the blank plasma, creating final concentrations of $0.003,0.013$ and $0.053 \mathrm{mmol} / 1$, respectively. Each sample was processed as described in the paragraph entitled 'Determination of the behaviors of derivatives releasing DHAP in vitro and preparation of plasma samples', apart from the fact that the derivative methanol solution was added and then determined by HPLC. Recovery rates were calculated according to the standard curve of DHAP or HPPE in plasma.

Three concentrations of the plasma solutions, namely $0.003,0.013$ and $0.053 \mathrm{mmol} / \mathrm{l}$, were prepared in triplicate and 
determined by two HPLC experiments performed in 1 day and then continuously for 5 days. The relative standard deviation (RSD) within-day and between days was subsequently calculated.

Method of administration, blood collection and processing. The rat i.g. DHAP and all 3 derivatives were the same as those aforementioned. The i.m. and intravenous (i.v.) doses of DHAP administered to the rats were 0.39 and $0.20 \mathrm{mmol} / \mathrm{kg}$, respectively. Following administration, $0.5 \mathrm{ml}$ blood were collected via the fundus venous plexus into a centrifuge tube containing $55 \mu \mathrm{l}$ of $3.8 \%(\mathrm{w} / \mathrm{v})$ sodium citrate solution at a series of time points (DHAP i.v.: 5.0, 17.0, 20.0, 25.0, 35.0 45.0, 60.0, 75.0, 90 and 120.0 min; DHAP i.m.: 1.0, 5.0, 10.0, 15.0, 20.0, 25.0, 30.0, 45.0, 60.0, 75.0, 90.0 and $120.0 \mathrm{~min}$; DHAP i.g.: 3.0, 5.0, 10.0, 18.0, 25.0, 29.0, 44.0, 60.0, 75.0 and $120.0 \mathrm{~min}$; APDP i.g., HPPE i.g. and PEG-DHAP i.g.: 0.05, 0.083, 0.17, 0.25, 0.5, 1.0, 2.0, 4.0, 6.0, $12.0,24.0$ and $48.0 \mathrm{~h}$ ). Samples were subsequently processed according to the aforementioned blood processing procedure, except for the addition of methanol solution of derivative.

Data processing. Drug and statistics software (DAS; version 2.1.1) provided by Shanghai Bojia Pharmaceutical Technology Co., Ltd. was used to process the data. Additionally, the pharmacokinetic parameters were calculated. The relative oral bioavailability of the derivatives $\left(\mathrm{F}_{\mathrm{R}} \%\right)$ was calculated using the following formula:

$$
F_{R} \%=\frac{A U C_{(0-t)}(\text { derivative }) \times \text { Dig, DHAP }}{A U C_{(0-t)}(\text { ig }, \text { DHAP }) \times D(\text { derivative })} \times 100 \%
$$

where D stands for the dose for i.g. administration.

\section{Results}

Synthesis of derivatives. APDP was prepared through the acylation of 3-hydroxyl and 4-hydroxyl via propionyl chloride. HPPE was prepared through the etherification of 4-hydroxyl via benzyl bromide. The polymer derivative, PEG-DHAP, was prepared by bonding DHAP to polyethylene glycol 2000 via ester bonds. The preparation process and conditions were simple and are presented in Fig. 1. The structural identification spectra of DHAP and the 3 derivatives are presented in Figs. S1-S4. The synthesis of the derivates was as follows.

APDP (yield, 90.3\%; purity, 97.6\%). ${ }^{1} \mathrm{H}$ NMR (DMSO- $d_{6}$, $600 \mathrm{MHz}): \delta 7.86$ (1H, dd, H-6), 7.8 (1H, d, H-2), 7.3 (1H, d, H-5), $2.60\left(7 \mathrm{H}, \mathrm{br}, \mathrm{O}=\mathrm{C}-\mathrm{CH}_{3},-\mathrm{CH}_{2}-\right), 1.27\left(6 \mathrm{H}, \mathrm{t},-\mathrm{CH}_{3}\right) ;{ }^{13} \mathrm{C} \mathrm{NMR}$ (DMSO- $\left.d_{6}, 150 \mathrm{MHz}\right)$ : $\delta 196.05(\mathrm{C}-7), 171.57(\mathrm{C}-12), 171.21$ (C-9), 146.1 (C-4), 142.33 (C-3), 135.47 (C-1), 126.75 (C-6), 123.68 (C-2), 123.62 (C-5), 27.50 (C-13), 27.41 (C-10), 26.55 (C-8), 9.09 (C-11), 9.03 (C-14); FTIR (KBr): 3,100.83-3,060.00 $\left(v_{=\mathrm{C}-\mathrm{H}}\right), 2,989.03-2,884.24\left(v_{\mathrm{C}-\mathrm{H}}\right), 1,764.53\left(v_{\mathrm{C}=\mathrm{O}, \text { ester }}\right), 1,683.37$ $\left(v_{\mathrm{C}=\mathrm{O} \text {,ketone }}\right), 1,601.74-1,462.28\left(v_{\mathrm{C}=\mathrm{C} \text {, aromatic ring }}\right), 1,421.65-1,318.23$ $\left(\delta_{\mathrm{C}-\mathrm{H}}\right), 1,279.26-1,174.37\left(v_{\mathrm{C}-\mathrm{O}-\mathrm{C}, \text { ester }}\right)$. EI-MS, $265.1072 \mathrm{~m} / \mathrm{z}$, $(\mathrm{M}+\mathrm{H})^{+}, \mathrm{C}_{14} \mathrm{H}_{17} \mathrm{O}_{5}$. mp $72.0-73.7^{\circ} \mathrm{C}$.

HPPE (yield, 70.1\%; purity, 99.1\%). ${ }^{1} \mathrm{H}$ NMR (DMSO-d, $600 \mathrm{MHz})$ : $\delta 9.42$ (1H, s, 3-OH), 7.49 (1H, dd, H-6), $7.42(5 \mathrm{H}$, br, H-11, H-12, H-13, H-14, H-15), 7.34 (1H, d, H-2), 7.09 (1H, d, H-5), $5.21\left(2 \mathrm{H}, \mathrm{s},-\mathrm{CH}_{2}-\right), 2.47\left(3 \mathrm{H}, \mathrm{s}, \mathrm{O}=\mathrm{C}-\mathrm{CH}_{3}\right) ;{ }^{13} \mathrm{C} \mathrm{NMR}$
(DMSO-d6, $150 \mathrm{MHz})$ : $\delta 196.83$ (C-7), 151.46 (C-4),147.17 (C-3), 137.23 (C-10), 130.83 (C-1), 128.87 (C-12, C14), 128.34 (C-13), 128.14 (C-11, C-15), 121.65 (C-6), 115.22 (C-2), 70.23 (C-9), $26.75(\mathrm{C}-8)$; FTIR (KBr): 3,173.39 $\left(v_{=\mathrm{C}-\mathrm{H}}\right), 1,653.42\left(v_{\mathrm{C}=\mathrm{O}}\right.$ ketone $), 1,605.93-1,428.72\left(v_{\mathrm{C}=\mathrm{C} \text {, aromatic ring }}\right), 1,276.96,1,211.98$ $\left(\delta_{\mathrm{C}-\mathrm{H}}\right), 1,141.52\left(v_{\mathrm{C}-\mathrm{O}-\mathrm{C}}\right)$. EI-MS, $243.1016 \mathrm{~m} / \mathrm{z},(\mathrm{M}+\mathrm{H})^{+}$, $\mathrm{C}_{15} \mathrm{H}_{15} \mathrm{O}_{3}$. mp $122.0-123.4^{\circ} \mathrm{C}$.

PEG-DHAP (yield, 52.7\%). ${ }^{1} \mathrm{H}$ NMR (DMSO- $d_{6}, 600 \mathrm{MHz}$ ): 86.57-8.24 (3-OH and protons of aromatic ring), 3.52 $\left(-\mathrm{OCH}_{2} \mathrm{CH}_{2} \mathrm{O}-\right)$; FTIR (KBr): 3,426.59 $\left(v_{\mathrm{OH}}\right), 2,880.85\left(v_{\mathrm{C}-\mathrm{H}}\right)$, $1,731.72\left(v_{\mathrm{C}=\mathrm{O}}\right), 1,588.12\left(v_{\mathrm{C}=\mathrm{C} \text {, aromatic ring }}\right), 1,467.53 \sim 1,242.20$ $\left(\delta_{\mathrm{C}-\mathrm{H}}\right), 1,113.34\left(v_{\mathrm{C}-\mathrm{O}-\mathrm{C}, \text { ester }}\right)$. DHAP content, $12.47 \%$.

Physicochemical properties of DHAP and its derivatives. The water solubility and oil-water partition coefficient (presented using $\log$ ) of compounds are presented in Table I. DHAP was found to be highly soluble in water, with a solubility of $176.68 \mathrm{mg} / \mathrm{ml}$. It also exhibited a low oil-water partition coefficient $(\log P=0.90)$, suggesting that it can be absorbed rapidly through epithelial cells, similarly to water-soluble propofol. APDP and HPPE exhibited markedly lower water solubilities and higher oil-water distribution coefficients compared with DHAP, due to possessing hydrophobic hydrocarbyl chains (Table I). Their LogP-values were in the range of 0-3, which is optimal for the penetration of orally administered drugs through the phospholipid biomembrane via passive diffusion (38). PEG-DHAP is highly water-soluble. The mean particle size of PEG-DHAP in its $0.5 \mathrm{mg} / \mathrm{l}$ aqueous solution was $260.90 \mathrm{~nm}$, suggesting that PEG-DHAP nanoparticles can be absorbed via epithelial endocytosis (39).

Derivative release of DHAP in vitro. Selecting an appropriate UV detection wavelength is critical for the simultaneous quantitative determination of derivatives and DHAP in plasma by HPLC. As presented in Fig. S5, the maximum absorption wavelengths of all 3 derivatives were at $246 \mathrm{~nm}$, with that of DHAP being $275 \mathrm{~nm}$. The absorption curves intersected at a wavelength of $260 \mathrm{~nm}$, they exhibited the same degree of absorption at $260 \mathrm{~nm}$. Therefore, $260 \mathrm{~nm}$ was selected as the detection wavelength for the simultaneous quantitative determination of the 3 DHAP derivatives.

As presented in the HPLC chromatograms depicted in Fig. 2, the retention times $\left(t_{R}\right)$ of DHAP, phenol (internal standard), APDP, HPPE and PEG-DHAP were 4.36, 6.05, $11.48,11.57$ and $2.74 \mathrm{~min}$, respectively. The peak area values of the main chromatographic peaks are shown in Table II. The 3 derivatives existed in different forms within in vitro plasma, as presented in Fig. 2E-G. A total of $89.40 \%$ of APDP transformed into DHAP (Fig. 2E), 7.90\% of PEG-DHAP released DHAP (Fig. 2G), and almost all HPPE existed as the prototype in plasma (Fig. 2F). The release of DHAP from APDP and PEG-DHAP may be due to the hydrolysis of ester bonds catalyzed by carboxylesterases in plasma. The hydrolysis of ether bonds in HPPE requires CYP-450, which is insufficient in plasma (40). Therefore, HPPE does not transform into DHAP in plasma in vitro.

Derivatives releasing DHAP in vivo. The present study assessed whether the derivatives released DHAP following 
Table I. Values of water solubility and $\log P$ of compounds ${ }^{\mathrm{a}}$.

\begin{tabular}{lcccc}
\hline Solubility/LogP & DHAP & APDP & HPPE & PEG-DHAP \\
\hline Water solubility $(\mathrm{mg} / \mathrm{ml})$ & $176.68 \pm 12.33$ & $0.23 \pm 0.02$ & $0.15 \pm 0.007$ & $48.33 \pm 3.73$ \\
LogP & $0.904 \pm 0.022$ & $1.538 \pm 0.063$ & $2.219 \pm 0.097$ & - \\
\hline
\end{tabular}

${ }^{a}$ The data shown are as the means \pm SD $(n=3)$. DHAP, 3,4-dihydroxyacetophenone; APDP, 4-acetyl-1,2-phenylene dipropionate; HPPE, 1-(3-hydroxy-4-phenoxy-phenyl)-ethanone.
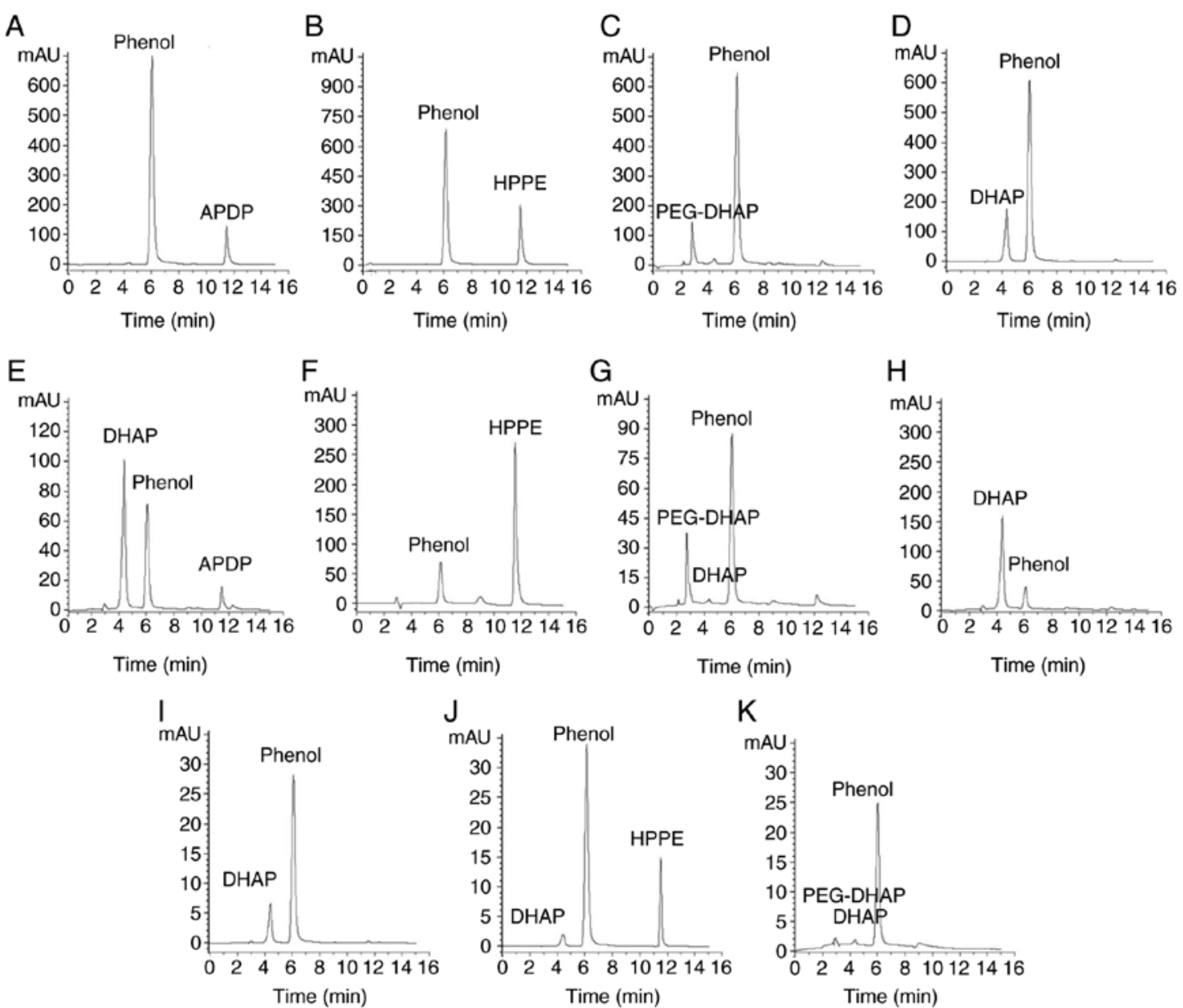

Figure 2. Behaviors of derivatives releasing DHAP in vitro and in vivo. HPLC chromatograms of APDP (A) in methanol, (E) in plasma in vitro and (I) in plasma in vivo; HPLC chromatograms of HPPE (B) in methanol, $(\mathrm{F})$ in plasma in vitro and (J) in plasma in vivo; HPLC chromatograms of PEG-DHAP (C) in methanol, $(\mathrm{G})$ in plasma in vitro and $(\mathrm{K})$ in plasma in vivo; HPLC chromatograms of DHAP (D) in methanol, $(\mathrm{H})$ in plasma in vitro. In in vitro experiments, $\mathrm{n}=3$; in in vivo experiments, $\mathrm{n}=6$. DHAP, 3,4-dihydroxyacetophenone; APDP, 4-acetyl-1,2-phenylene dipropionate; HPPE, 1-(3-hydroxy-4-phenoxy-phenyl)-ethanone.

i.g. administration to rats to determine which form was active in vivo and which form may be a target for blood concentration determination. When comparing the derivative HPLC chromatograms in methanol (Fig. 2A-C) and in plasma following i.g. administration (Fig. 2I-K), all APDP and a quantity of HPPE and PEG-DHAP transformed into or released DHAP $\sim 10$ min after the administration. For PEG-DHAP, only the free DHAP transported across the PEG barriers was active. Therefore, DHAP is the active form of both APDP and PEG-DHAP in plasma after absorption and distribution. For HPPE, the original form and DHAP produced by metabolism were present in the blood, synchronously, $10 \mathrm{~min}$ after oral administration. Whether the remaining HPPE may be used as the active substance for determining blood concentrations depends on whether it is active or not.

Antiplatelet aggregation activity. If HPPE itself exerts antiplatelet aggregation activity, it is necessary to determine the blood concentration of HPPE. Therefore, the in vitro antiplatelet aggregation activity of DHAP and its derivatives was determined.

DHAP exhibited antiplatelet aggregation activity. Its in vitro activity was concentration-dependent, as presented in Fig. 3A, and the half-maximal inhibitory concentration $\left(\mathrm{IC}_{50}\right)$ was $99.22 \mu \mathrm{mol} / 1$. The $\mathrm{IC}_{50}$ of APDP was 
Table II. Values of the HPLC chromatography peak area in study of the behaviors of derivatives releasing DHAP in vitro and in vivo ${ }^{\mathrm{a}}$.

\begin{tabular}{|c|c|c|c|c|c|}
\hline & Phenol & DHAP & APDP & HPPE & PEG-DHAP \\
\hline APDP in methanol ${ }^{\mathrm{b}}$ & $12,561.5 \pm 345.6$ & - & $1,897.2 \pm 117.9$ & - & - \\
\hline HPPE in methanol ${ }^{\mathrm{b}}$ & $11,862.3 \pm 218.7$ & - & - & $2,837.8 \pm 163.6$ & - \\
\hline PEG-DHAP in methanol ${ }^{\mathrm{b}}$ & $11,511.2 \pm 316.2$ & - & - & - & $1,764.8 \pm 110.1$ \\
\hline DHAP in methanol ${ }^{\mathrm{b}}$ & $10,636.7 \pm 228.4$ & $2,023.3 \pm 89.2$ & - & - & - \\
\hline APDP in plasma in vitro ${ }^{\mathrm{b}}$ & $1,594.2 \pm 115.2$ & $2,225.1 \pm 179.3$ & $263.7 \pm 18.3$ & - & - \\
\hline HPPE in plasma in vitro ${ }^{\mathrm{b}}$ & $1,562.5 \pm 127.5$ & - & - & $5,254.3 \pm 213.6$ & - \\
\hline PEG-DHAP in plasma in vitro ${ }^{\mathrm{b}}$ & $1,448.0 \pm 137.4$ & $36 \pm$ & - & - & $419.6 \pm 40.8$ \\
\hline DHAP in plasma in vitro ${ }^{\mathrm{b}}$ & $1,525.7 \pm 72.7$ & $7,039.0 \pm 229.5$ & - & - & - \\
\hline APDP in plasma in vivo ${ }^{\mathrm{c}}$ & $648.6 \pm 47.5$ & $177.0 \pm 8.4$ & - & - & - \\
\hline HPPE in plasma in vivo ${ }^{\mathrm{c}}$ & $718.0 \pm 50.5$ & $43.3 \pm 2.1$ & - & $269.8 \pm 19.4$ & - \\
\hline PEG-DHAP in plasma in vivo ${ }^{\mathrm{c}}$ & $542.8 \pm 32.7$ & $15.9 \pm 1.3$ & - & - & $22.1 \pm 1.8$ \\
\hline
\end{tabular}

${ }^{a}$ The data shown are as the means $\pm \mathrm{SD} ;{ }^{b} \mathrm{n}=3 ;{ }^{\mathrm{c}} \mathrm{n}=6$. DHAP, 3,4-dihydroxyacetophenone; APDP, 4-acetyl-1,2-phenylene dipropionate; HPPE, 1-(3-hydroxy-4-phenoxy-phenyl)-ethanone.
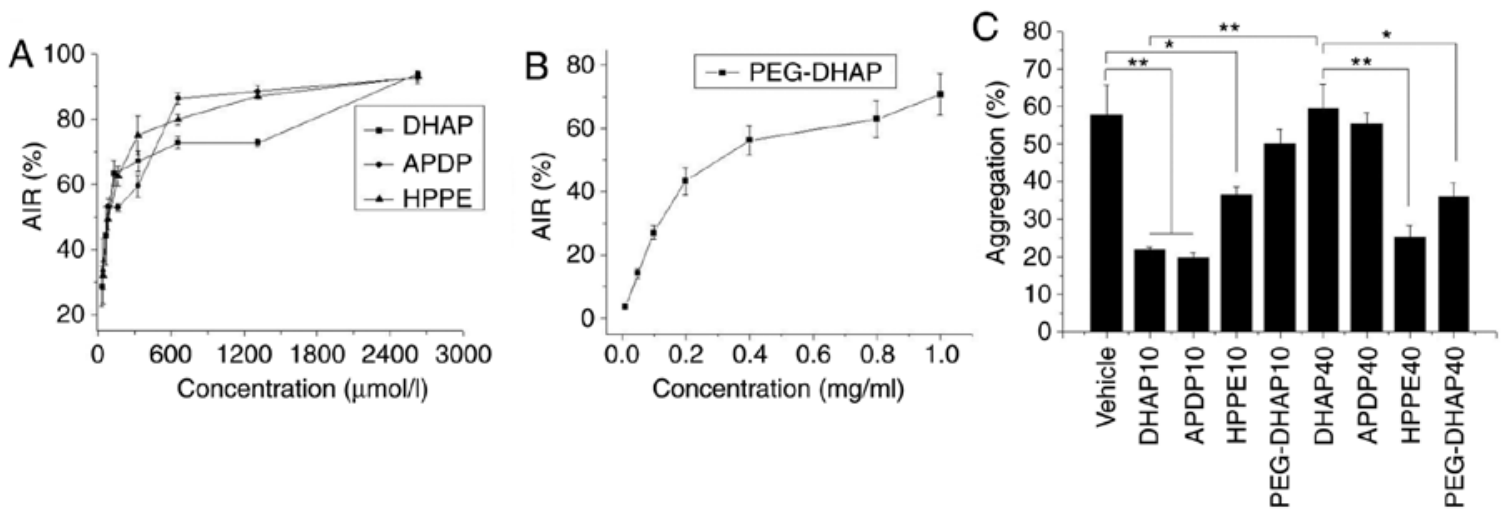

Figure 3. Antiplatelet aggregation activity (A) AIR of DHAP, APDP and HPPE in vitro (B) AIR of PEG-DHAP in vitro (C) AR of DHAP and derivatives in vivo. The data are presented as the means $\pm \mathrm{SD}$. ${ }^{*} \mathrm{P}<0.05$ and ${ }^{* *} \mathrm{P}<0.01$, with comparisons indicated by lines. DHAP10, APDP10, HPPE10 and PEG-DHAP10, indicate $10 \mathrm{~min}$ after the administration of the compound; DHAP40, APDP40, HPPE40 and PEG-DHAP40, indicate 40 min after the administration of the compound. In in vitro experiments each drug concentration group was independently determined 4 times. In in vivo experiments used 6 rats in each group, and the blood of each rat was independently determined 4 times. DHAP, 3,4-dihydroxyacetophenone; APDP, 4-acetyl-1,2-phenylene dipropionate; HPPE, 1-(3-hydroxy-4-phenoxy-phenyl)-ethanone.

$104.59 \mu \mathrm{mol} / 1$, which was almost equal to that of DHAP. This result may be due to the transformation of almost all APDP into DHAP following incubation in plasma for $5 \mathrm{~min}$. The $\mathrm{IC}_{50}$ of HPPE was $92.35 \mu \mathrm{mol} / 1$, which was also similar to DHAP. Since HPPE cannot be cleaved into DHAP in in vitro plasma (Fig. $2 \mathrm{~F}$ ), the results indicated that HPPE itself exhibited antiplatelet aggregation activity, similar to DHAP. HPPE may therefore be used as one of the active substances for the determination of blood concentration. The $\mathrm{IC}_{50}$ of PEG-DHAP was $0.25 \mathrm{mg} / \mathrm{ml}$, which was equivalent to $173.20 \mu \mathrm{mol} / 1$ of DHAP (Fig. 3B). The higher $\mathrm{IC}_{50}$ value demonstrated herein may be due to the slow diffusion of DHAP across PEG barriers, a notion that implies the possibility of slow release DHAP.

In vivo antiplatelet aggregation measurements were performed in the current study to further assess the in vivo processes and the activity of the 3 derivatives. As presented in Fig. 3C, DHAP $(\mathrm{P}=0.003)$ and APDP $(\mathrm{P}=0.002)$ significantly inhibit platelet aggregation after $10 \mathrm{~min}$ of i.g. administration compared with the vehicle, indicating that they functioned rapidly in vivo. Following $10 \mathrm{~min}$ of i.g. administration, HPPE and PEG-DHAP exhibited a lower antiplatelet aggregation activity and a slower action time compared to DHAP. After 40 min of i.g. administration, the antiplatelet aggregation activity of DHAP was significantly reduced compared with 10 min of administration $(\mathrm{P}=0.003)$, and APDP exhibited very weak activities, while HPPE $(\mathrm{P}=0.006)$ and $\mathrm{PEG}-\mathrm{DHAP}(\mathrm{P}=0.021)$ demonstrated significant activity compared with 40 min of administration of DHAP. The results confirmed that DHAP has a short acting time in vivo and is rapidly adsorbed and eliminated, which is in congruence with previously reported data $(13,16)$. APDP rapidly transformed into DHAP in vivo, thereby allowing for rapid elimination. The long-lasting activity of HPPE and PEG-DHAP imply that the rapid metabolism of DHAP may be delayed by HPPE and PEG-DHAP, indicating their 
Table III. Parameters used to evaluate the method of blood concentration determination ${ }^{\mathrm{a}}$.

\begin{tabular}{lccccc}
\hline Compound & $\begin{array}{c}\text { Concentration } \\
(\mathrm{mmol} / \mathrm{l})\end{array}$ & Recovery $(\%)^{\mathrm{b}}$ & $\begin{array}{c}\text { Within-day } \\
\text { RSD }(\%)\end{array}$ & $\begin{array}{c}\text { Between-days } \\
\text { RSD }(\%)\end{array}$ & $\begin{array}{c}\text { Storage } \\
\text { stability }^{\mathrm{b}}(\%)\end{array}$ \\
\hline DHAP & 0.003 & $86.00 \pm 26.21$ & 3.84 & 14.07 & $84.12 \pm 12.25$ \\
& 0.013 & $105.67 \pm 3.93$ & 1.42 & 15.29 & $88.85 \pm 8.42$ \\
HPPE & 0.053 & $101.52 \pm 1.10$ & 2.64 & 5.36 & $95.00 \pm 3.61$ \\
& 0.003 & $92.24 \pm 17.02$ & 4.27 & 5.99 & $96.76 \pm 4.47$ \\
& 0.013 & $98.77 \pm 4.41$ & 2.58 & 3.17 & $96.81 \pm 6.23$ \\
& 0.053 & $104.62 \pm 2.29$ & 1.12 & 1.15 & $98.47 \pm 3.20$ \\
\hline
\end{tabular}

${ }^{\mathrm{a}} \mathrm{n}=3$; ${ }^{\mathrm{b}}$ data shown are the means \pm SD. DHAP, 3,4-dihydroxyacetophenone; APDP, 4-acetyl-1,2-phenylene dipropionate; HPPE, 1-(3-hydroxy-4-phenoxy-phenyl)-ethanone.
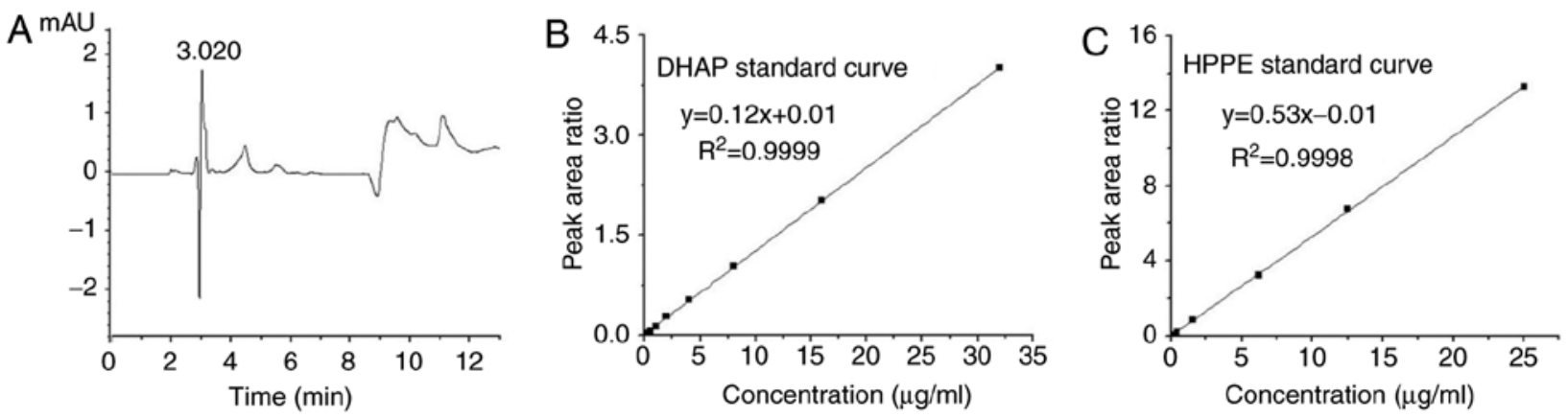

Figure 4. Assessments of the HPLC analytical methods of the blood concentration determination (A) HPLC chromatogram of blank plasma, (B) standard curve of DHAP in plasma, (C) standard curve of HPPE in plasma. DHAP, 3,4-dihydroxyacetophenone; APDP, 4-acetyl-1,2-phenylene dipropionate; HPPE, 1-(3-hydroxy-4-phenoxy-phenyl)-ethanone.

ability to prolong acting time in vivo and improve the oral bioavailability of DHAP.

Assessments of HPLC analytical method. Phenol was selected as the internal standard. Under the HPLC conditions selected for the determination of blood concentration, the target substances were well separated from phenol and were not interfered with by the endogenous substances of plasma (Figs. 2 and 4A). DHAP was the active substance for the determination of APDP and PEG-DHAP blood concentrations, and HPPE and DHAP were the active substances for the determination of HPPE blood concentrations. Therefore, the standard curves of DHAP and HPPE were determined. Within the range of $0.10-2.00 \mu \mathrm{g} / \mathrm{ml}$, the association between the plasma concentration of DHAP, $x$, and the peak area ratio (DHAP vs. internal standard), $y$, was linear. The linear regression equation was, $R^{2}=0.9999$ (Fig. 4B). In the range of $0.10-25.00 \mu \mathrm{g} / \mathrm{ml}$, the association between the plasma concentration of HPPE, $x$, and the peak area ratio (HPPE vs. internal standard), $y$, was linear. The linear regression equation was, $R^{2}=0.9998$ (Fig. 4C). As presented in Table III, the values of recovery of the high, medium and low concentrations of plasma DHAP and HPPE were in the range of 86.00-105.67 and 92.24-104.62\%, respectively. Within-day precision was presented using RSD and was in the range of $1.42-3.84$ and $1.12-4.27 \%$, respectively. The between-day precision of RSD was in the range of 5.36-15.29 and $1.15-5.99 \%$, respectively. Additionally, solution storage stability was within the range of 84.12-95.00 and 96.76-98.47\%, respectively. The results revealed that the analytical method used for the determination of blood concentrations met the requirements of pharmacokinetic studies.

Blood concentration and bioavailability. In the present study, the pharmacokinetic parameters of DHAP administered i.v. to rats were consistent with a two-compartment model, which was same as reported previously $(33,34)$. The values of distribution half-life $\mathrm{t}_{1 / 2(\alpha)}$ and elimination half-life $\mathrm{t}_{1 / 2(\beta)}$ were 0.045 and $0.282 \mathrm{~h}$, respectively. Due to the rapid distribution and elimination of DHAP when administered i.v., according to a previous study (41), a single-compartment model simulation was used to compare with other groups. The pharmacokinetic parameters of the other drug-administered groups were consistent with the single-compartment model, as presented in Table IV. The mean blood concentration-time curves are presented in Fig. 5.

As presented in Fig. 5A, DHAP exhibited rapid absorption and rapid elimination rates after i.v., i.m. and i.g. administration in rats, as reported in previous studies involving monkeys and rabbits $(33,34)$. It can be detected in plasma within 3 min after i.m. and i.g. administration. The mean residence time in the body $\left(\mathrm{MRT}_{(0-\mathrm{t})}\right)$ was within the range of $0.22-0.46 \mathrm{~h}$, and the elimination half-life $\left(\mathrm{t}_{1 / 2}\right)$, was in the range of $0.15-0.16 \mathrm{~h}$. Its very short in vivo acting time and rapid elimination rate may be due to the severe first pass effect that was confirmed by the markedly higher bioavailability of i.m. administration relative to i.g. administration (Table IV). The severe first pass 
Table IV. Pharmacokinetic parameters of the tested compounds ${ }^{\mathrm{a}}$.

\begin{tabular}{lccccccc}
\hline Parameter & Unit & DHAP i.v. & DHAP i.m. & DHAP i.g. & APDP & HPPE & PEG-DHAP \\
\hline$t_{1 / 2}{ }^{d}$ & $\mathrm{~h}$ & $0.15 \pm 0.02$ & $0.16 \pm 0.015$ & $0.16 \pm 0.02$ & $0.13 \pm 0.01$ & $1.06 \pm 0.14$ & $1.80 \pm 0.27$ \\
$K_{e}{ }^{d}$ & $1 / \mathrm{h}$ & $4.81 \pm 0.73$ & $4.36 \pm 0.383$ & $4.27 \pm 0.41$ & $5.35 \pm 0.34$ & $0.66 \pm 0.08$ & $0.39 \pm 0.05$ \\
$V_{l} / F^{d}$ & $1 / \mathrm{kg}$ & $0.48 \pm 0.05$ & $0.71 \pm 0.07$ & $4.03 \pm 0.83$ & $2.49 \pm 0.63$ & $6.97 \pm 2.17$ & $13.48 \pm 4.08$ \\
$C L / F^{d}$ & $1 / \mathrm{h} / \mathrm{kg}$ & $2.30 \pm 0.38$ & $3.121 \pm 0.56$ & $17.39 \pm 4.81$ & $13.35 \pm 3.56$ & $4.70 \pm 1.78$ & $5.36 \pm 2.05$ \\
$A U C(0-t)^{d}$ & $\mu \mathrm{mol} / \mathrm{l} \mathrm{h} \mathrm{h}$ & $66.32 \pm 1.67$ & $124.22 \pm 3.50$ & $47.78 \pm 2.11$ & $34.59 \pm 2.29$ & $118.06 \pm 15.04$ & $6.69 \pm 4.35$ \\
$\mathrm{AUC}(0-\infty)^{\mathrm{d}}$ & $\mu \mathrm{mol} / \mathrm{l} \mathrm{h}$ & $87.48 \pm 2.02$ & $129.94 \pm 3.66$ & $48.64 \pm 2.18$ & $35.94 \pm 2.48$ & $123.26 \pm 15.54$ & $7.03 \pm 4.79$ \\
$\mathrm{~K}_{\mathrm{a}}{ }^{\mathrm{d}}$ & $1 / \mathrm{h}$ & & $5.13 \pm 0.64$ & $11.77 \pm 1.40$ & $6.43 \pm 0.56$ & $12.01 \pm 2.43$ & $0.44 \pm 0.06$ \\
$\mathrm{t}_{1 / 2} \mathrm{~K}_{\mathrm{a}}{ }^{\mathrm{d}}$ & $\mathrm{h}$ & & $0.14 \pm 0.02$ & $0.06 \pm 0.01$ & $0.11 \pm 0.01$ & $0.06 \pm 0.01$ & $1.62 \pm 0.25$ \\
$\mathrm{Tlag}^{\mathrm{d}}$ & $\mathrm{h}$ & & $0.04 \pm 0.01$ & $0.066 \pm 0.00$ & $0.04 \pm 0.00$ & $0.04 \pm 0.00$ & $0.03 \pm 0.03$ \\
$\mathrm{MRT}^{\mathrm{d}}(0-\mathrm{t})^{\mathrm{e}}$ & $\mathrm{h}$ & $0.22 \pm 0.03$ & $0.46 \pm 0.01$ & $0.38 \pm 0.02$ & $0.36 \pm 0.01$ & $1.35 \pm 0.24$ & $4.36 \pm 0.28$ \\
$F \%$ & & & $93.81^{\mathrm{b}}$ & $18.01^{\mathrm{b}}$ & $126.14^{\mathrm{c}}$ & $394.78^{\mathrm{c}}$ & $425.44^{\mathrm{c}}$
\end{tabular}

${ }^{\mathrm{a}} \mathrm{n}=6$. he data shown are the means $\pm \mathrm{SD}$; ${ }^{b}$ absolute bioavailability; ${ }^{\mathrm{c}} \mathrm{FR} \%$, relative bioavailability relative to DHAP i.g. administration; ${ }^{\mathrm{d}}$ calculated form a single-compartment model; ${ }^{\mathrm{c}}$ calculated from statistical moment model. DHAP, 3,4-dihydroxyacetophenone; APDP, 4-acetyl-1,2-phenylene dipropionate; HPPE, 1-(3-hydroxy-4-phenoxy-phenyl)-ethanone.
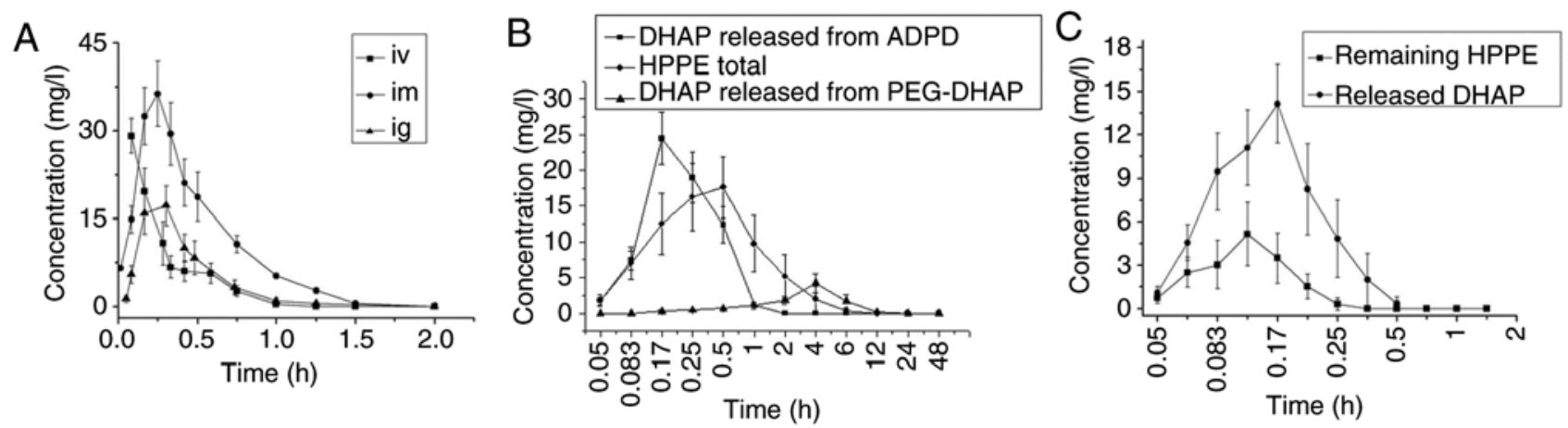

Figure 5. The average blood concentration-time curves of (A) DHAP after i.v., i.m. and i.g. administration, (B) derivatives after i.g., and (C) remaining HPPE and released DHAP after i.g. administration in rats. $n=6$. The data are presented as the means \pm SD.

effect may also be confirmed via the i.v.-clearance value listed in Table III of $2.30 \mathrm{l} / \mathrm{h} / \mathrm{kg}$, which was greater than the liver blood flow rate of the rats. This indicated that DHAP exhibited both hepatic and extrahepatic metabolisms.

Compared with DHAP, APDP exhibited a much lower water solubility and a higher oil-water partition coefficient and was therefore easier to pass through the biofilm via passive diffusion, making its absorption rate faster. As presented in Table IV, its MRT ${ }_{(0-\mathrm{t})}, \mathrm{t}_{1 / 2}$ and $F_{R} \%$ were $0.36,0.13 \mathrm{~h}$ and $126.14 \%$, respectively. However, no marked difference from the DHAP i.g. group was observed. For HPPE, the average blood concentration-time curves of its original form and DHAP form produced by metabolism are presented in Fig. 5C. Its pharmacokinetic parameters were calculated using total the concentration of HPPE and DHAP. Its MRT $\mathrm{MR- \textrm {t }}, \mathrm{t}_{1 / 2}$ and $F_{R}$ was $1.35,1.06 \mathrm{~h}$ and $394.78 \%$, respectively. The results were different to those of the DHAP i.g. group. HPPE therefore prolongs the in vivo acting time and half-life of DHAP and improves its oral bioavailability. For PEG-DHAP, as presented in Table IV, its value of $\mathrm{MRT}_{(0-\mathrm{t})}$ was $4.36 \mathrm{~h}$, which was 11.47 -fold higher than that of DHAP. Its value of $\mathrm{t}_{1 / 2}$ was also $1.80 \mathrm{~h}$, which was 11.88-fold higher than that of DHAP. Finally, its value of $F_{R} \%$ was $425.44 \%$. A prolonged in vivo acting time and an improved oral bioavailability of PEG-DHAP were obtained.

\section{Discussion}

The phenolic hydroxyl groups of catechol compounds are essential for biological activity. They are also the main sites of metabolic inactivation (35). Modifying the phenolic hydroxyls of DHAP may impact its associated pharmacokinetic processes and oral bioavailability. Fortunately, these phenolic hydroxyls can be modified easily and there are many simple and classic chemical reactions available for reference and selection. In the present study, the esterification or etherification of the phenolic hydroxyl groups located at $\mathrm{C} 3$ or $\mathrm{C} 4$ were selected as the generated derivatives as they may be cleavage catalyzed by enzymes in vivo to recover DHAP. Furthermore, the synthetic methods that obtained high yields utilized in the present study can be implemented in any laboratory.

DHAP is a water-soluble small molecule that is absorbed rapidly through epithelial cells in the present study, similarly to the water soluble propofol. DHAP was also eliminated rapidly regardless of the route of administration and exhibited a severe 
first pass effect, which was in congruence with other studies on monkeys and rabbits $(33,34)$. The derivatives produced in the current study exhibited different physicochemical properties, biological activities or in vivo processes compared with DHAP. APDP was produced as a derivative with two propionate groups at $\mathrm{C} 1$ and $\mathrm{C} 2$. These hydrophobic carbon chains caused APDP to possess a much lower water solubility and much higher values of $\log P$, resulting in a more rapid absorption rate compared with DHAP. However, APDP exhibited no significant differences in antiplatelet activity, in vivo process and oral bioavailability when compared with DHAP. Although APDP was a derivative obtained by modifying the main metabolic sites of DHAP, its modified ester bonds were rapidly hydrolyzed in intestinal cells and the liver during absorption, which recovered the hydroxyls and lost the protection of the metabolic sites. This was also confirmed by the results of the derivative-releasing DHAP experiments, which revealed that plasma APDP recovers to DHAP rapidly, whether in vivo or in vitro. Therefore, this did not prolong the in vivo acting time and half-life of DHAP or improve its oral bioavailability. Although HPPE was also a derivative obtained via the derivatization of the phenolic hydroxyl group of DHAP, the introduced hydrophobic carbon chain was attached to the phenyl ring via an ether bond and thus it exerted different antiplatelet aggregation activities and in vivo processes from APDP. HPPE is an aryl ether compound, and its metabolic pathway involves the $\mathrm{O}$-dealkylation reaction, which transpires within the liver. In this, the carbon-oxygen bond is cleaved to produce a phenol group. Although the rate of this metabolism is very rapid, HPPE tends to accumulate in the lipid layer of biofilms, releasing slowly into bodily fluids due to its low water solubility and high oil-water partition coefficient. Therefore, HPPE transformed into DHAP at a slower rate relative to ADPD. The average blood concentration-time curves of HPPE (Fig. 5C) and the derivative-releasing DHAP experiments revealed that a portion of HPPE transformed into DHAP in vivo, a short time after absorption. However, some still remained within the blood and DHAP was gradually released with an extended administration time. HPPE itself also exhibited significant antiplatelet aggregation activity. During a certain administration time, the activity was contributed to by both the released DHAP and the remaining HPPE. Therefore, the kinetic parameters of HPPE were calculated in the present study based on the total concentrations of DHAP and HPPE in the blood. The significantly longer in vivo time, higher relative oral bioavailability and longer-lasting antiplatelet aggregation activity after oral absorption confirmed that HPPE improves the clinical application defects of DHAP. PEG-DHAP is a polymer derivative obtained by linking DHAP to crosslinked PEG 2000 via ester bonds. Its dose and the unit of measurement were not same as those of DHAP, APDP and HPPE. PEG-DHAP is a high molecular drug, and the molecular weight is not very accurate. Therefore, the dose unit was expressed by the mass of the weighing. In order to facilitate a comparison with DHAP and two other small molecular prodrugs, we calculated the moles of DHAP contained in the dosage of PEG-DHAP. The lower dose of PEG-DHAP was selected as the same dose of PEG-DHAP was dissolved in $1.5 \mathrm{ml}$ of normal saline to form a very viscous solution, which could not guarantee the normal administration of intragastric administration. The effective results of several animal experiments were obtained at these doses i) in vivo release after administration only to determine out whether the drug can release DHAP; ii) determination of IC50 of in vivo antiplatelet aggregation; and iii) pharmacokinetic experiments). PEG-DHAP disperses into nanoparticles within bodily fluids and is adsorbed via the phagocytosis of epithelial cells (39). Although ester bonds can be rapidly hydrolyzed and catalyzed by carboxylesterases present in the blood, cytosol and endoplasmic reticulum, it takes some time for esterases and associated metabolizing enzymes to cross the PEG barrier and come into contact with DHAP. Additionally, it takes some time for free DHAP to migrate out of the PEG barrier to reach sites at which it may exhibit pharmacological activity. Therefore, for PEG-DHAP, a prolonged in vivo acting time and improved oral bioavailability were obtained.

In the present study, other metabolites of DHAP, including sulfate, gluconate metabolites were not considered. Previous pharmacodynamic studies have demonstrated that DHAP exhibits the strongest antiplatelet aggregation activity following 10 min of i.g. administration, after which the activity continues to decrease, and disappears completely after $40 \mathrm{~min}(13,16)$. Pharmacokinetics also revealed that DHAP reaches a peak plasma concentration at 5-10 min after i.g. administration, after which rapid metabolism is observed, and plasma DHAP is absent after $30-40$ min $(33,34)$. DHAP has a catechol structure and is rapidly metabolized in vivo to gluconate and sulfate metabolites. Combined with the pharmacokinetic and pharmacodynamics results of previous studies, it can be inferred that these gluconate and sulfate metabolites have no antiplatelet aggregation activity. Therefore, only the concentrations of possible active substances in blood were determined in the present study after ig administration in rats. DHAP in blood was measured during the blood concentration determination of APDP and PEG-DHAP, and both concentrations of blood HPPE and DHAP were measured in the pharmacokinetic study of HPPE.

DHAP is a water-soluble small molecule that exhibits severe first pass metabolism, which is the reason for its short acting time in vivo and relatively low oral bioavailability. By modifying the metabolic sites of DHAP, three derivatives, including APDP, HPPE and PEG-DHAP, were prepared in the current study to alter the pharmacokinetics of DHAP. These derivatives transformed into or released DHAP in vivo. Among these, HPPE and PEG-DHAP significantly increased the acting time in vivo and the oral bioavailability of DHAP. The results of the current study may provide a possible reference point for the development of DHAP as an oral drug. Furthermore, these results may be useful for researchers to address the poor bioavailability of phenolic drugs.

\section{Acknowledgements}

The authors would like to thank Dr Bo Zhang and Dr Xuejian Wang from the School of Pharmacy, Weifang Medical College for their helpful assistance with the calculation of pharmacokinetic parameters.

\section{Funding}

The study was supported by the National Natural Science Foundation of China (grant no. 31600386), the Natural Science Foundation of Shandong Province (grant no.ZR2016HM47) and 
the Medical and Health Science and Technology Development Project of Shandong Province (grant no. 2014WSB27002).

\section{Availability of data and materials}

All data generated or analyzed during this study are included in this published article.

\section{Authors' contributions}

NS and YD were responsible for the synthesis of derivatives and pharmacokinetic experiments. MQ and YL were responsible for the determination of physicochemical properties and platelet aggregation experiments. MW and LW were responsible for raising the animals and treating the animals used in the experiments. All authors have read and approved the final manuscript.

\section{Ethics approval and consent to participate}

All procedures performed in experiments involving animals were approved by the Animal Experimentation Ethics Committee of Weifang Medical College and in accordance with the guidelines of the National Health and Medical Research Council of China for the care and use of animals for scientific purposes.

\section{Patient consent for publication}

Not applicable.

\section{Competing interests}

The authors declare that they have no competing interests.

\section{References}

1. Beijing Pharmaceutical Industry Research Institute, Chinese People's Liberation Army 157 Hospital: Study on the active constituents of leaves of Ilex pubescens Hook et Arn var glaber Chang. Chin Trad and Herb Drugs Commun 8: 7-10, 1977 (In Chinese).

2. Research Group of the Therapeutic Effects of Qingxintong: Summary of the treatment effects of Qingxintong on angina pectoris of coronary heart disease. Beijing Pharm Industry 1: 23-27, 1983 (In Chinese).

3. Chinese People's Liberation Army 157 Hospital: Clinical observation of 50 cases of coronary heart disease treated with Qingxintong injection. Beijing Pharm Industry 1: 27-29, 1983 (In Chinese).

4. Beijing pharmaceutical industry research institute: Effect of Qingxintong on thrombosis in patients with coronary heart disease. Beijing Pharm Industry 1: 16-17, 1983 (In Chinese).

5. Beijing Pharmaceutical Research Institute: Research on the pharmacologic effect of leaves of Qingxintong on coronary heart disease. Chin Trad and Herb Drugs 11: 358-366, 1980 (In Chinese).

6. Lin CL, Zhang ZX and Xu YJ: Therapeutic mechanism of qingxintong on chronic obstructive pulmonary disease. Chin J Tuberculosis and Respirat 18: 97-98, 1995 (In Chinese).

7. Lin CL, Zhang ZX, Xu YJ and Ni W: Effects of qingxintong on hemodynamics and atrial natriuretic peptide and cyclic glucosides in patients with chronic obstructive pulmonary disease Chin J Integrated Trad Chin Western Med 15: 131-133, 1995 (In Chinese).

8. Lin CL, Zhang ZX and Xu YJ: Effects of 3.4-dihydroxyacetophenine on hemorheology and plasma levels of TXA2, CD62P in patients with chronic pulmonary heart disease. J Clin Internal Med 19: 196-198, 2002 (In Chinese).
9. Sun YP, Ma TY and Wu XR: Clinical analysis and mechanism of treatment of pregnancy-induced hypertension with Qingxintong. Practical J Obstet Gynecol 7: 141-143, 1991 (In Chinese).

10. Huang YP and Ma TY: Treatment of intrauterine growth retardation with qingxintong. Chin J Obstet Gynecol 28: 333-336, 1993 (In Chinese).

11. Wu XR, Li Y, Yang DS, Han BJ, Si YZ, Shan ZZ, Ma TY, Wen LZ, Sun YP and Huang YP: The utero-placental circulation, eugenics and the prevention and treatment of high risk pregnancies. J Tongji Med Univ 14: 1-7, 1994.

12. Huang YP, Ye DY, Wu P, Huang YF, Ying HG and Ma TY: The therapeutic effectiveness of inhaled DHAP in the treatment of intrauterine growth retardation. Acta Med Univ Sci Et Technol Huazhong 31: 192-194, 2002 (In Chinese).

13. Wang Z, Gao HQ, An Y, Zhu GQ and Yang ZM: Effect of 3,4-dihydroxyacetophenone on rabbit platelet function. Zhongguo Yao Li Xue Bao 5: 187-192, 1984 (In Chinese).

14. Wang Z, Gao HQ, Zhu GQ, An Y and Huang RS: Effect of 3,4-dihydroxyacetophenone on the generation of PGI2-like substances in the rat aorta. Zhongguo Yao Li Xue Bao 7: 37-40, 1986 (In Chinese).

15. $\mathrm{Xu} \mathrm{H}$, Wang $\mathrm{Z}$ and $\mathrm{An} \mathrm{Y:} \mathrm{Effect} \mathrm{of} \mathrm{3,4-dihydroxyacetophenone}$ on platelet phosphodiesterase in rabbits. Acta Pharmacol Sin 8: 159-162, 1986 (In Chinese).

16. Wang Z, An Y, Liu Z, Zhu GQ and Huang RS: Effect of 3,4-dihydroxyacetophenone on TXA2 release from rabbit platelets. Yao Xue Xue Bao 22: 330-334, 1987 (In Chinese).

17. $\mathrm{Li} \mathrm{S}, \mathrm{Li} \mathrm{Y}$, Xiong $\mathrm{Z}$ and $\mathrm{Wu} \mathrm{X}$ : Influence of Qingxingtong on platelet function of PIH-patients. J Tongji Med Univ 23: 305-307, 1994 (In Chinese).

18. Wang Z, Huang RS, Gao YH, An Y and Qiang ZG: 3,4-Dihydroxyacetophenone-a cyclooxygenase inhibitor (brief). Acta Pharmacol Sin 1: 35, 1988 (In Chinese).

19. Shi L, Qin ZH and Gao SX: Effect of 3,4-dihydroxyacetophenone on the membrane fluidity of platelets by fluorescence polarization analysis. Zhongguo Yao Li Xue Bao 7: 149-151, 1986 (In Chinese).

20. Shan $\mathrm{Z}$, Chang $\mathrm{C}$, Feng $\mathrm{L}$ and $\mathrm{Wu} X$ : The regulatory effect of 3,4-dihydroxyacetophenone on tPA and PAI activity of the vascular endothelial calls. Pharmacol Clin Chin Materia Med 6: 33-35, 1995 (In Chinese).

21. Beijing Institute of Pharmaceutical Industry, The People's Liberation Army 157 Hospital: Pharmacological study of bald holly leaves on coronary heart disease. Chin Pharm J 15: 42, 1980 (In Chinese).

22. Wu P, Huang YP and Ye DJ: Advances in research on the mechanism of qingxintong promoting blood circulation and removing blood stasis. Chin Trad Herbal Drugs 23: 277-279, 2001 (In Chinese).

23. Ullah F, Wang DX, Ming Z and Yu SB: Effects of 3,4-dihydroxyacetophenone (3,4-DHAP) on hypoxic pulmonary and systemic vascular response in dogs. J Tongji Med Univ 15: 26-30, 1995.

24. Hong ZG, Wang DX, Jin S, Zhang J, Wang XL and Ye DY: Effect of 3,4-dihydroxyacetophenone on delayed rectifier potassium current of the intrapulmonary artery smooth muscle cells in rats. Chin Pharmacol Bull 18: 386-389, 2002 (In Chinese).

25. Yang DS, Xi-rui W and Ting-yuan M: Effects of 3,4-dihydroxyacetophenone on the biosynthesis of TXA2 and PGI2 in human placental villus and umbilical artery segments in vitro. Prostaglandins 38: 497-504, 1989.

26. Hang YP, Ye DJ and Ma TJ: Effect of Qingxintong on nitric oxide synthase and plasma endothelin in placental vascular wall of patients with pregnancy induced hypertension. Chin J Obstet Gynecol 31: 667-669, 1996 (In Chinese).

27. Li G, Wu P, Zhang DJ, Ye DY and Li F: Mechanism of inhibition of 3,4-dihydroxyacetophenone on LPS-induced apoptosis of RAW264.7 macrophages in mice. Chin Trad Herbal Drugs 36: 1835-1838, 2005 (In Chinese).

28. Wu P, Zhang L, Zhou X, Li Y, Zhang D, Wan J and Ye D: Inflammation pro-resolving potential of 3,4-dihydroxyacetophenone through 15 -deoxy- $\Delta 12,14$-prostaglandin $\mathrm{J} 2$ in murine macrophages. Int Immunopharmacol 7: 1450-1459, 2007.

29. Zhang D, Liu T, Liu J, Cui X, Guo J, Wang J and Ye D: Relationship between therapeutic and preventive effect of dihydroxyacetophenone in treating atherosclerosis and the Toll-like-receptor 4 pathway. Trad Chin Drug Res Clin Pharmacol 20: 404-407, 2009 (In Chinese).

30. Zhang D, Liu T, Cui X, Liu J, Guo J, Wang J and Ye D: Preventive effect of 3,4-dihydroxyacetophenone on atherosclerosis and role of visfatin expression. Chin J Pathophysiol 26: 1700-1703, 2010 (In Chinese). 
31. Zhang D, Gong Y, Liu J, Wang J, Wang L and Liu T: Effects of 3,4-dihydroxyacetophenone on 5-lipoxygenase in macrophages of atherosclerosis plaque. Trad Chin Drug Res Clin Pharmacol 23: 243-246, 2012 (In Chinese).

32. Zhang DJ, Liu JY, Wang L, Wang J, Li W, Zhuang B, Hou J and Liu T: Effects of 3,4-dihydroxyacetophenone on the hypercholesterolemia-induced atherosclerotic rabbits. Biol Pharm Bull 36: 733-740, 2013

33. Li SX, Li Y, Shan ZZ, Xiong ZM, Wu XR and Yin XG: Pharmacokinetics of Qingxintong in rabbits. Acta Universitatis Medictnae Tangji 22: 91-93, 1993 (In Chinese).

34. Pang X, Fu G and Zuo M: Comparison of bioavailability of Qingxintong by three administration routes. Trad Chin Drug Res Clin Pharmacol 13: 31-32, 2001 (In Chinese)

35. Eun JK, Jong WA, Hye SL and Wolfram C: Determination of peripheral catecholO-methyltransferase (COMT) activity in vivo using $\left(2-{ }^{14} \mathrm{C}\right)-3^{\prime}, 4^{\prime}-$ dihyroxyacetophenone. Arch Pharm Res 14: 290-294, 1991

36. Montenegro L, Carbone C, Maniscalco C, Lambusta D, Nicolosi G, Ventura CA and Puglisi G: In vitro evaluation of quercetin-3-O acyl esters as topical prodrugs. Int J Pharm 336 257-262, 2007.
37. Jin YY, Zhang JS, Zhang Y and Zhang YH: Studies on the intestinal adsorption of crocin in rats and determination of the partition coefficient. J Chin Pharm Univ 35: 247-257, 2004 (In Chinese).

38. Fang L: Pharmaceutics. 8th edition. People's Medical Publishing House, Beijing, pp137-139, 2016 (In Chinese).

39. Zhou X, Zhang X, Han S, Dou Y, Liu M, Zhang L, Guo J, Shi Q, Gong G, Wang R, et al: Yeast microcapsule-mediated targeted delivery of diverse nanoparticles for imaging and therapy via the oral route. Nano Lett 17: 1056-1064, 2017.

40. You QD: Medicinal Chemistry. 8th edition. People's Medical Publishing House, Beijing, p46, 2016 (In Chinese).

41. Liu CX: Introduction to Pharmacokinetics. Liu D (ed) China Academic Press Publication, Beijing, pp215-217, 1984 (In Chinese). 